\title{
UNSTEADY FLOWFIELD AROUND TANDEM CYLINDERS AS PROTOTYPE FOR COMPONENT INTERACTION IN AIRFRAME NOISE
}

\author{
Mehdi R. Khorrami* ${ }^{*}$ Meelan M. Choudhari ${ }^{*}$, Luther N. Jenkins**, and Catherine B. McGinley** \\ NASA Langley Research Center \\ MS 128, Hampton, VA
}

\begin{abstract}
Synergistic application of experiments and numerical simulations is crucial to understanding the underlying physics of airframe noise sources. The current effort is aimed at characterizing the details of the flow interaction between two cylinders in a tandem configuration. This setup is viewed to be representative of several component-level flow interactions that occur when air flows over the main landing gear of large civil transports. Interactions of this type are likely to have a significant impact on the noise radiation associated with the aircraft undercarriage. The paper is focused on two-dimensional, time-accurate flow simulations for the tandem cylinder configuration. Results of the unsteady Reynolds Averaged Navier-Stokes (URANS) computations with a two-equation turbulence model, at a Reynolds number of 0.166 million and a Mach number of 0.166 , are presented. The experimental measurements of the same flow field are discussed in a separate paper by Jenkins, Khorrami, Choudhari, and McGinley (2005). Two distinct flow regimes of interest, associated with short and intermediate separation distances between the two cylinders, are considered. Emphasis is placed on understanding both time averaged and unsteady flow features between the two cylinders and in the wake of the rear cylinder. Predicted mean flow quantities and vortex shedding frequencies show reasonable agreement with the measured data for both cylinder spacings. Computations for short separation distance indicate decay of flow unsteadiness with time, which is not unphysical; however, the predicted sensitivity of mean lift coefficient to small angles of attack explains the asymmetric flowfield observed during the experiments.
\end{abstract}

\section{Introduction}

Airframe noise constitutes a major component of the total aircraft noise during approach and landing. For medium and large civil transports, a significant portion of the airframe noise can be attributed to the landing gears and in particular the main landing gear. The proximity of many bluff bodies of various sizes

\footnotetext{
*Aerospace Technologist, Computational Aerosciences Branch, Associate Fellow AIAA

** Aerospace Technologist, Flow Physics and Control Branch
} 
and shapes that collectively make up the main landing gear, produce an extremely complex flow field that is highly interactive and nonlinear in nature. Physics-based modeling of landing-gear noise requires a fundamental understanding of the unsteady flow interactions between the various components. A canonical configuration that models a variety of component level interactions is that of multiple cylinders with axes parallel to each other. Cylinder configurations such as multiple wheels, axles, and brake pistons include cylinders of similar size, whereas the combination of main strut and the hydraulic lines involves interaction between cylinders of disparate scales. The present paper is focused on the case of similar size tandem cylinders. Configurations of cylinders of disparate diameters are currently under consideration and will be reported in future papers.

Although the case of a tandem cylinder configuration has been considered before ${ }^{1}$, nearly all of the past studies were restricted to the low Reynolds (Re) number flow regime ${ }^{1-3}$, which is dominated by laminar flow separation and periodic vortex shedding. According to reference 2, several distinct flow regimes have been observed in such flows. The three most prominent correspond to short, intermediate, and large separation distances between the cylinders' centerlines. In the case of short separations (L/D < 2.4), the two cylinders behave as a single bluff body with vortex shedding occurring at the rear cylinder only. For large separation distances, the flow field approaches that of isolated single cylinders. At intermediate separation distances, the flow is bi-stable, switching intermittently between the flow states corresponding to short and large separation distances. This critical flow regime is the most difficult to simulate computationally.

Despite their usefulness, the earlier studies lack detailed on-surface and off-surface measurements that are needed for benchmarking the computations and enabling physics-based models for sound radiation. The present computational effort is part of a synergistic combination of experiments and flow simulations that is aimed at providing a holistic view of the tandem cylinder flow field at sufficiently large Reynolds numbers. Separation distances of $\mathrm{L} / \mathrm{D}=1.435$, and 3.7 (representing two of the three possible flow regimes observed in earlier experiments) were chosen for this joint study. The experimental effort was conducted in the Basic Aerodynamic Research Tunnel (BART) at LaRC. The ease of optical access at BART allowed detailed measurements of the flow field using Particle Image Velocimetry (PIV) technique. Moreover, extensive measurements in the wake of the rear cylinder were conducted using single and dual hot-wire probes to obtain the frequency content and two-point correlations within the unsteady flow field. 
The experiments were conducted at a Reynolds number of $\mathrm{Re}=166,000$ based on the cylinder diameter and the maximum tunnel speed of $M=0.166$. To ensure turbulent flow separation, the boundary layer on the front cylinder was tripped between azimuthal locations of 50 and 60 degrees on either side of the leading stagnation point. The measured surface pressure distribution for the large separation case was nearly identical to that measured by previous investigators for a single, isolated cylinder at a Reynolds number greater than 8 million. Details of the measurements conducted in BART are discussed in the companion paper by Jenkins, Khorrami, Choudhari, and McGinley $^{4}$ (2005, here after referred to as $\mathrm{JKCM})$.

\section{Computational Procedure}

\section{Flow Solver}

The CFL3D code developed at NASA Langley Research Center (LaRC) has been used to compute the flow field. Based on a finite-volume formulation, CFL3D solves the compressible form of three-dimensional, time-dependent, thin-layer Navier-Stokes equations. For the URANS computations, the 2-equation SST $(k-\omega)$ model of Menter $^{2}$ is used.

All current computations are performed using the second-order-accurate time discretization and the “dual time stepping" method. ${ }^{3}$ Twenty subiterations, in conjunction with 3-level V-type multigrid cycles, are utilized to ensure a minimum of two orders of magnitude drop in both the mean-flow and turbulencemodel residuals during each time step.

\section{Geometry and Grid Topology}

The geometry under consideration is comprised of two cylinders of equal diameter (D) aligned along the streamwise direction (tandem configuration) as shown in figure 1 . The diameter of each cylinder is 1.75 inches $(0.04445 \mathrm{~m})$. The cylinders span the entire tunnel height, $b$, of 28 inches $(0.71 \mathrm{~m})$, yielding an aspect ratio of $\mathrm{b} / \mathrm{D}=16$. Based on the tunnel width of 40 inches $(1.016 \mathrm{~m})$, the resulting model blockage (less than 4.4\%) is deemed to be small enough to minimize the effects of tunnel wall interference. A more detailed account of the model hardware and its setup in BART is provided by JKCM. Of the three distinct separation distances (between the cylinders' centerline) of $\mathrm{L} / \mathrm{D}=1.435,3.7$, and 7.0 emphasized during the measurements by JKCM, only the cases of 1.435 and 3.7 are simulated numerically. 
The global and near-field views of the grid topology for the $\mathrm{L} / \mathrm{D}=3.7$ case are displayed in figure 2. Rather than simulating the actual test section in BART, the chosen computational domain is for a freefield flow in order to remove reflection of the acoustic waves from the tunnel walls and thus, facilitating the computation of far-field noise radiation in the future. The placement of the outer boundaries is a minimum of thirteen diameters from the nearest point on the solid surfaces. Consistent with the objective behind this work, the computational grid is fine enough to resolve the dominant $2 \mathrm{D}$ scales of motion in between the two cylinders and in the wake of the rear cylinder. However, as explained in the next section, there are regions of the flow field that remain under-resolved and, hence, may contribute significantly to the observed discrepancies between the measurements and computations.

For the $\mathrm{L} / \mathrm{D}=3.7$ case, the computational domain surrounding the cylinders is divided into 11 blocks, involving significant mesh clustering near the solid surfaces and in the cylinders' wakes. The structured and patched two-dimensional (2D) grid contains a total of $319 \mathrm{~K}$ grid points.

Lessons learned from the $\mathrm{L} / \mathrm{D}=3.7$ solutions were applied towards several subtle but never-the-less important refinements of the grid for the $\mathrm{L} / \mathrm{D}=1.435$ configuration. The most prominent refinement involved achieving better spatial resolution in the wake of the rear cylinder. For the $\mathrm{L} / \mathrm{D}=1.435$ grid, the location of the outer boundaries remains identical to the previous case. The structured and patched 2D grid contains a total of $316 \mathrm{~K}$ points distributed into 16 blocks. Notice that the number of grid points in the two cases remains close, pointing to the fact that grid savings associated with the smaller gap between the two cylinders were utilized elsewhere to achieve the desired refinements.

\section{Results}

We now discuss the results of the numerical simulations; normalized with respect to the cylinder diameter $\mathrm{D}$ as the reference length, reference speed $U_{\infty}$, a reference density, $\rho_{\infty}$, and a reference molecular viscosity, $\mu_{\infty}$. For the present study, the reference flow variables were set to match the conditions at the entrance to the BART test section. The flow field is assumed to be fully turbulent from the start. The unsteady computations are based on the two equation $k$ - $\omega$ turbulence model ${ }^{5}$. The flow conditions for the simulations correspond to $\mathrm{M}=0.166$ and $\mathrm{Re}=1.66 \times 10^{5}$. However, limited additional results are also

presented for a $\operatorname{Re}=1.66 \times 10^{6}$. A constant non-dimensional time step of $\Delta t=0.00845$ (corresponding to 150 points per period for a $1000 \mathrm{~Hz}$ signal) is used throughout all simulations.

As expected, simulations for the $\mathrm{L} / \mathrm{D}=3.7$ case showed prominent vortex shedding from both cylinders. After the initial transient, the flow field settled down into a quasi-periodic state with lift 
coefficients (root-mean-square) and shedding frequencies that were in line with available data ${ }^{7}$ for high Reynolds number cylinder flows. The unsteady flow structures for the $\mathrm{L} / \mathrm{D}=1.435$ configuration turned out to be less energetic and the fluctuation intensity was gradually damped out following the initial transient phase. We begin the discussion with time-averaged quantities for each of the two configurations, followed by a comparison of the instantaneous flow fields with the experiment.

\section{$\underline{\text { Mean Surface Pressures }}$}

The time averaged surface pressure coefficient, $\mathrm{Cp}$, for the $\mathrm{L} / \mathrm{D}=3.7$ case is presented in figure 3 . Distributions for both front and rear cylinders are displayed. As a partial measure of grid independence, results from the medium-level grid (whereby every other point in both directions was omitted from the finest grid) are also plotted on the figure. Notice that the mid- and fine-level Cps are virtually indistinguishable. Overall, the computed $\mathrm{Cp}$ displays similar behavior as the measured pressure. Both computation and measurement show a distribution that is symmetric with two prominent suction peaks. Effect of the boundary-layer trips on the front cylinder (near $\theta=50$ and $\theta=310$ degrees, respectively) is clearly visible for the measured $\mathrm{Cp}$ distribution. The computation over-predicts the magnitude and predicts location of the suction peaks further downstream on the front cylinder and under-predicts the magnitude of the pressure recovery towards the back portion of the cylinder. The excessive dip in the pressure recovery near $\theta=180$ degrees has been observed in previous work related to a single cylinder (Vatsa and Singer 2003) ${ }^{8}$ and is generally attributed to the lack of three dimensional effects within the computation. On the contrary, the magnitude and location of the suction peaks are under-predicted for the rear cylinder, but good agreement is attained for the pressure recovery in the separated region. The observed discrepancies between 315 and 360 degrees may be due to a slight misalignment between the two cylinders. This will be discussed in the next section.

The $\mathrm{Cp}$ results for the case of $\mathrm{L} / \mathrm{D}=1.435$ are plotted in figure 4. For the initial comparison, focus on the two curves labeled "CFD" (solid line) and "Measurement" (symbol line). For the front cylinder (figure 4a), the computed pressures are in good agreement with the measurement. The magnitude and locations of the suction peaks and the separation points are well predicted and so is the magnitude of the pressure recovery in the back. Contrary to both single cylinder and tandem configurations with larger separations, the CFD result moderately over-predicts the base pressure behind the front cylinder at $\mathrm{L} / \mathrm{D}=$ 1.435. The reason for this over-prediction is not clear at this stage. Notice that there is a slight asymmetry to the measured $\mathrm{Cp}$. For the rear cylinder (figure $4 \mathrm{~b}$ ), the high pressure peak does not occur along the $\theta=$ 0 line. Two separate high- pressure peaks are observed at $\theta \approx \pm 50$ degrees, close to where the shear layers separating from the front cylinder impinge on the rear cylinder. The computed pressure shows the 
relevant trends but does not predict the measured amplitudes for these peaks. Most significantly, the mean CFD-based pressures are nominally symmetric about $\theta=0$, whereas a pronounced asymmetry is noted in the measured $\mathrm{Cp}$ distribution along the surface of the rear cylinder. Such a strong pressure asymmetry on the rear cylinder has also been observed by previous investigators ${ }^{9}$ but, to the best of our knowledge, no clear explanations for its occurrence have been provided as yet.

During the course of the experiments by JKCM, particular care was exercised to ensure nearly symmetric pressure distributions along both the front and the rear cylinders. While for larger separation distances it was relatively easy to achieve a symmetric $\mathrm{Cp}$ distribution, the pressure field became extremely sensitive to even minor perturbations for L/D less than 1.5. On certain occasions, it was possible to achieve close to symmetric pressure distributions, while routine (and seemingly minor) changes during the course of the testing phase resulted in a loss of symmetry at small separation distances. During the post-experiment discussions, the possibility that the presence of small irregularities whether in the misalignments of the fabricated model, model set-up in the tunnel, or incoming flow angularities could cause the asymmetry was raised. In particular, the effects of incoming flow angularity was deemed to be most critical since ultimately geometrical misalignments would also manifest themselves as a change in the incoming flow direction. It was further conjectured that minor incoming flow angularities would have less of an impact once the separation distance between the two cylinders is large enough to allow full blown shedding from each cylinder to be established. To test these hypotheses, a series of $2 \mathrm{D}$ simulations were carried out whereby the approaching inflow was at a nonzero but small angle-of-attack (AOA). Going back to figure 3, notice that an AOA of 1 degree has hardly any influence on the predicted surface pressure distribution at $\mathrm{L} / \mathrm{D}=3.7$ and all the computed $\mathrm{Cp}$ curves are virtually identical. In contrast, for the $\mathrm{L} / \mathrm{D}=1.435$ case, even a small change in the incoming flow direction produces large surface pressure asymmetries on the rear cylinder that are in line with the measured trends. Figure 5 shows the effect of inflow angularity on the total lift coefficient $\mathrm{C}_{\mathrm{L}}$. Observe that imparting a vertical velocity that is less than two percent of the freestream velocity results in a mean lift coefficient of approximately 0.16. A more challenging question is whether and how such small imperfections can be eliminated from an experimental setup. Surely, most entrance flows to any tunnel test section involve a small amount of flow angularity. Similar asymmetry also occurs naturally in complex flow configurations such as an aircraft undercarriage

\section{Time Averaged Off-surface Quantities}

The mean streamlines for the $\mathrm{L} / \mathrm{D}=3.7$ case are shown in figure 6 . The experimental streamlines are obtained from averaging over 1000 PIV snapshots. The plots provide useful insight into the extent of 
flow separation behind each cylinder. Contrary to the measurements, the computations predict that the larger of the two (mean) separation zones occurs behind the rear cylinder. One possible reason for this difference could be a lack of both sufficiently strong unsteady flow structures and 3D effects in the computation. In the experiment, fluid trapped within the recirculating zone can get pumped out by the weak spanwise flow along the base of the cylinder. Certainly, such effects are not captured by our 2D simulations. However, the observed differences for the separation zone of the front cylinder are somewhat more difficult to explain. Also note a slight asymmetry in the measured streamlines between the cylinders, pointing to the presence of a small flow angularity as discussed earlier.

The corresponding averaged streamwise and vertical velocity contours plus the spanwise vorticity field for the region in between the two cylinders are plotted in figure 7. As suspected, the computed velocity contours show a narrower recirculation zone compared with PIV data, along with a more rapid recovery in the wake deficit within the gap region. The slower recovery in the measured wake result in mean velocities in the region ahead of the rear cylinder that are on the order of $20 \%$ to $30 \%$ of $U_{\infty}$. The computed vertical velocities show the proper trends but tend to be larger than measured values, pointing to a more prominent roll-up (shedding) process. The computed vorticity contours diffuse very rapidly at much closer distances to the solid surface than indicated by the measurements. To adequately capture the evolution of the curved shear layer at such distances from the cylinder surface will require a much higher spatial resolution than that of the present computations.

For aeroacoustic modeling purposes, an in-depth knowledge of the fluctuating flow field is of paramount importance. The root mean square (rms) velocity fluctuations and the corresponding turbulent kinetic energy (TKE) plots for $\mathrm{L} / \mathrm{D}=3.7$ are displayed in figure 8 . While the overall patterns are captured correctly, the computations tend to significantly over-predict the magnitude of the perturbations. There could be several explanations for this over-prediction, starting with the $2 \mathrm{D}$ nature of our simulations along with intrinsic limitations of using conventional turbulence models for time accurate simulations. The second explanation involves the fact that vortex shedding process in the simulations is a single-frequency (i.e., artificially regular) event that tends to be more intense compared with the actual vortex roll-up and shedding processes. The latter (as will be seen in the next section), are much more diffused, involving interactions among a variety of scales.

The mean streamline patterns for $\mathrm{L} / \mathrm{D}=1.435$ are shown in figure 9 . For this case, the computed length $(\approx 1.4 \mathrm{D})$ of the separation zone behind the rear cylinder agrees better with the measurement than the 3.7 case does. Notice that the computed streamlines are symmetric, showing a dual structure pattern within the gap region. In contrast, the measured streamlines show a single recirculating zone with fluid 
particles moving in the counter-clockwise direction. Most likely, the asymmetric gap pattern in the experiment is established by a combination of incoming flow angularity and a nonzero spanwise flow towards the tunnel's ceiling and floor. For the 2D simulations however, once the detached shear layers from the front cylinder reattach to the rear cylinder, the fluid particles within the gap zone are trapped without the possibility of escaping the zone. On the other hand, the computed results show the dualstructure streamline pattern even when the angle of attack has a nozero value.

Contour plots of the mean velocities and the spanwise vorticity for $\mathrm{L} / \mathrm{D}=1.435$ case at zero AOA are presented in figure 10 and show reasonable agreement between the predicted and measured data. Also observe that the direction of the computed mean vertical velocity (figure 10c) within the gap region is opposite to that observed for the 3.7 configuration (figure 7c). Although, reversal of the velocity direction is understandable (due to the presence of the dual recirculating structures), the magnitudes of the peak velocities are a bit surprising. According to literature, a reduced gap should result in progressively stagnant flow within this zone. Notice that the measurements also show comparable velocity magnitudes within the gap albeit a different structure. But as discussed in JKCM, the gap region possesses a relatively quiescent perturbation field and therefore aeroacoustically speaking is not of much relevance. The spanwise vorticity field (figure 10e) clearly displays the formation of the free-shear-layers and their detachment locations from the front cylinder. The shear layers reattach to the rear cylinder without going through a typical roll-up process thus, reinforcing the fact that for short gaps, the tandem cylinders act as a single bluff body. As alluded to in the earlier sections, after the initial transient state, the $\mathrm{L} / \mathrm{D}=1.435$ computations revealed a decaying time-dependent flow field with rapidly diminishing fluctuating fields. Naturally, the discussion of the perturbation fields is omitted. It suffice to say that the PIV and hot-wire measurements (see JKCM) all indicate the fluctuating fields to be much less energetic than the corresponding fields for $\mathrm{L} / \mathrm{D}=3.7$.

\section{Time-Dependent Flow field}

A sample plot of the instantaneous spanwise vorticity field from the $\mathrm{L} / \mathrm{D}=3.7$ simulation is shown in figure 11. Important features such as boundary layer separation, shear layer formation, roll-up, and formation of discrete vortices are clearly depicted in this figure. The vorticity field shows the convection and interaction of the vortices with the rear cylinder. The stagnation point on the front of the rear cylinder exhibits large excursions about the zero degree line due to this interaction. Also note the large undulation of the shear layers emanating from the rear cylinder before the roll-up process takes place. As indicated earlier, the simulated shedding process shows an intense event whereby the detached vorticity rolls up into large scale vortices scaling with the cylinder diameter. In contrast, the PIV snapshot in figure $11 \mathrm{~b}$ 
shows a much more benign roll-up process that is comprised of a large number of structures with a smaller scale. Additional noteworthy features of the PIV snapshot include the presence of smaller rollers residing within the detached shear-layer and the amalgamation of these rollers into a large structure that resembles a typical shed vortex. Note that the smaller rollers maintain their individual identity even up to the time they reach the rear cylinder. The smaller vorticity lumps in figure $12 \mathrm{~b}$ are the result of KelvinHelmholtz (K-H) instabilities of the free shear layer, which represent the local large scale motion of the flow and are known to scale with the shear-layer thickness. The presence of K-H instabilities in a cylinder wake was first observed by Couregelongue ${ }^{10}$ as early as 1929 . Figure 12a, which is reproduced from Zdravkovich $^{7}$, shows a schematic depiction of wake structures based on Couregelongue's observation of the flow field at $1200<\operatorname{Re}<4000$. The smaller vortices riding on the mixing layers were called "transition eddies" by these investigators. A sample of our PIV snapshot that depicts similar flow topology is also shown in figure 12b. In their PIV study of tandem cylinders at Re $=10,000$, Lin et al. ${ }^{11}$ also observed K-H instabilities and the accompanying convective rollers. The measurements by JKCM at $\mathrm{Re}=0.166$ million indicate that the dynamics of K-H instabilities and its effects on the wake structure can be observed over a large range of Reynolds numbers.

For the sake of completeness, a PIV snapshot of the instantaneous vorticity field for L/D = 1.435 is displayed in figure 13. The plot shows the growth of K-H instabilities (analogous to the L/D = 3.7 case) and the resulting chain of vortices that are convected downstream.

JKCM used hot wire measurements to obtain frequency spectra of velocity fluctuations for both $\mathrm{L} / \mathrm{D}=3.7$ and $L / D=1.435$. The measured spectra at a location 2.7D downstream and 0.69D below the axis of the rear cylinder are shown in figure 14. For reference, the figure includes the hot wire spectrum for the single cylinder case at the same location relative to the cylinder axis. The peak frequency and the corresponding Strouhal number, St, are also indicated for each curve. The spectrum for L/D = 3.7 possesses a distinct narrow-band peak that is an order of magnitude above the surrounding frequencies. The spectrum for $\mathrm{L} / \mathrm{D}=1.435$ displays a significantly lower but broader peak that contains approximately $60 \%$ of the energy compared with the peak at $\mathrm{L} / \mathrm{D}=3.7$ (or, equivalently, $39 \%$ of the energy contained within the peak for the single cylinder case). It is not clear if this change in the character of the Strouhal shedding is somehow related to the lack of self-sustaning unsteady structures within the CFD solution.

The time history of the computed total lift coefficient for $\mathrm{L} / \mathrm{D}=3.7$ shows a periodic state with a frequency of approximately $314 \mathrm{~Hz}$. This value is close to the measured frequency of $303 \mathrm{~Hz}$, although one must bear in mind that the hot wire spectrum is based on local velocity fluctuations at a single point. Additional analysis of the numerical database is necessary before a direct comparison can be made 
between the predicted and measured spectra. The predicted lift coefficient for the L/D = 1.435 case maintains a weak periodic state while the oscillations are being damped in time, as seen from figure 5. The frequency of these oscillations is close to $323 \mathrm{~Hz}$, which is not too far from the measured value of $353 \mathrm{~Hz}$.

\section{$\underline{\text { Reynolds Number Effects }}$}

An important issue when it comes to cylinder flow is the effects of Re on the state of the wake. Given the excellent agreement between our medium-grid and fine-grid solutions at $\operatorname{Re}=0.166$ million, it was decided that the fine grid might also be adequate for unsteady calculations at a higher Reynolds number. We repeated the $\mathrm{L} / \mathrm{D}=3.7$ simulation for $\mathrm{Re}=1.66$ million while keeping the Mach number constant. The predicted surface $\mathrm{Cp}$ distributions at the higher Re are plotted in figure 15 . The higher Re tends to improve the pressure recovery along the leeward side, but worsens the windward suction peaks along the front cylinder. Also, boundary layer separation locations are moved significantly aft of the locations measured for JKCM's tripped configuration. On the contrary, the Cp distribution for the rear cylinder shows significantly improved agreement with the measured pressures. The magnitude and location of the suction peaks as well as the base pressure recovery are consistent with measurements. Of course, computations at even higher Reynolds numbers (and commensurately finer grids) are required to assess the convergence of surface pressures across an adequately wide Reynolds number band. Additionally, the effects of Re on the off-surface flow field also need to be evaluated.

\section{Conclusion}

A combined computational and experimental effort is directed towards understanding highly interactive flow fields between multiple bluff bodies that are representative of flows encountered in main landing gears. A pair of cylinders in tandem configuration is a canonical geometry that occurs often in main gears. The computations are focused on 2D simulations of the flow past a tandem configuration for separation distances of $\mathrm{L} / \mathrm{D}=1.435$ and 3.7, representing small and medium gaps, respectively. For $\mathrm{L} / \mathrm{D}=$ 3.7, vortex shedding occurs at both cylinders and a periodic global state is established within the URANS computations. The computed shedding frequency based on the oscillations in the global lift is $314 \mathrm{~Hz}$, which agrees quite well with the experimentally measured frequency of $303 \mathrm{~Hz}$. The character of the flow field changes dramatically when the gap is reduced to 1.435 . The shear layers detaching from the front cylinder reattach to the rear cylinder surface, effectively creating a single bluff body. However, for $\mathrm{L} / \mathrm{D}=$ 1.435, the computations are unable to sustain the flow unsteadiness and thus the fluctuating field gets damped over time. Whether or not the predicted weakness of fluctuations at $\mathrm{L} / \mathrm{D}=1.435$ is related to the 
diffuse nature of the Strouhal peak in the measured hot wire spectrum remains to be determined. The sensitivity of the CFD solution to slight changes in angle of attack at short separation distances explains the occurrence of asymmetric mean flow in the wind tunnel experiment of JKCM, despite exercising due precaution to achieve a symmetric mean flow.

Instantaneous vorticity fields based on the PIV measurements indicate highly dynamic wakes at both short and intermediate separation gaps. PIV data also reveals the prominence of smaller scale vortex structures that arise as Kelvin-Helmholtz instabilities within shear layer regions. The current URANS computations and the grid distributions are incapable of capturing the full wake dynamics as observed in the PIV snapshots.

\section{Acknowledgment}

The authors would like to express their appreciation to Mr. M.R. Wiese of the Analytical Services and Materials, Inc. for generating the grid used during the computations.

\section{References}

1. Zdravkovich, M.M., "Review of Flow Interference Between Two Circular Cylinders in Various Arrangements,” J. Fluids Engineering, Vol., pp. 618-633, December 1977.

2. Zdravkovich, M.M., "Flow Induced Oscillations of Two Interfering Circular Cylinders," J. of Sound and Vibrations, Vol. 101, No.4, pp. 511-521, 1985.

3. Sun, J., Li, J., and Roux, B., "Flow Regimes and Frequency Selection of a Cylinder Oscillating in an Upstream Cylinder Wake,” International J. for Numerical Methods in Fluids, Vol. 16, pp. 915-929, 1993

4. Jenkins, L.N., Khorrami, M.R., Choudhari, M.M., and McGinley, C.B., "Characterization of Unsteady Flow Structures Around Tandem Cylinders for Component Interaction Studies in Airframe Noise," AIAA paper AIAA-2005-2812, May 2005.

5. Menter, F., "Improved Two-Equation k- $\omega$ Turbulence Models for Aerodynamic Flows," NASA TM 103975, 1992.

6. Rumsey, C. L., Sanetrik, M. D., Biedron, R. T., Melson, N. D., and Parlette, E. B., "Efficiency and Accuracy of Time-Accurate Turbulent Navier-Stokes Computations," Computers \& Fluids, Vol. 25, No. 2, pp. 217-236, 1996. 
7. Zdravkovich, M.M., "Flow Around Circular Cylinders-Vol 1: Fundamentals," Published by Oxford University Press, New York, 1997.

8. Vatsa, V.N. and Singer, B.A., "Evaluation of a Second-Order Accurate Navier-Stokes Code for Detached Eddy Simulation Past a Circular Cylinder,” AIAA paper AIAA-2003-4085, June, 2003.

9. Gu, Z., "On the Interference between Two Circular Cylinders at Supercritical Reynolds Number," J. of Wind Engineering and Industrial Aerodynamics, Vol. 62, pp. 175-190, 1996.

10. Couregelongue, J., "On the Existence of two Families of Eddies behind Immersed Solids," (in French). Comptes Rendus Academie Sciences, Vol. 189, pp. 972-974, 1929.

11. Lin, J.-C., Yang, Y., and Rockwell, D., "Flow Past Two Cylinders in Tandem: Instantaneous and Average Flow Structure,” J. of Fluids and Structures, Vol. 16, Issue 8, pp. 1059-1071, 2002. 

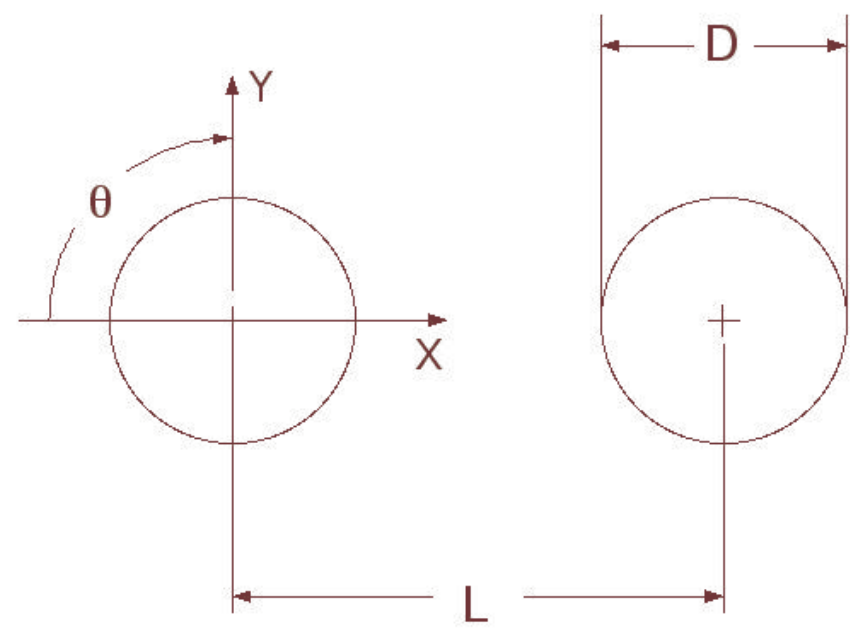

Fig.1. Schematic diagram of simulated configuration

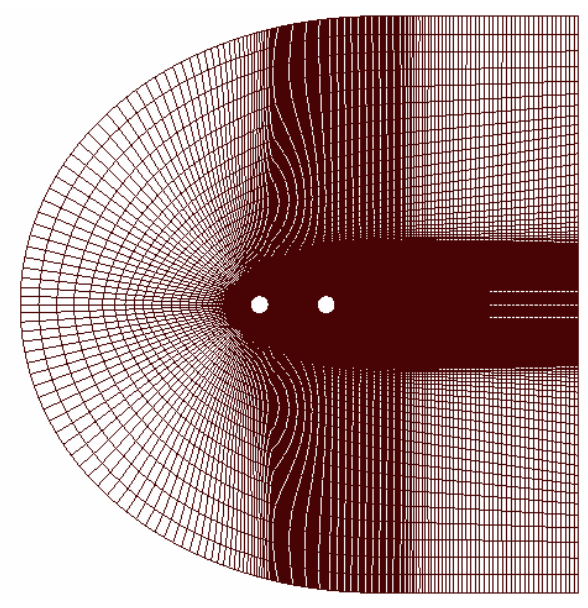

a) Full domain

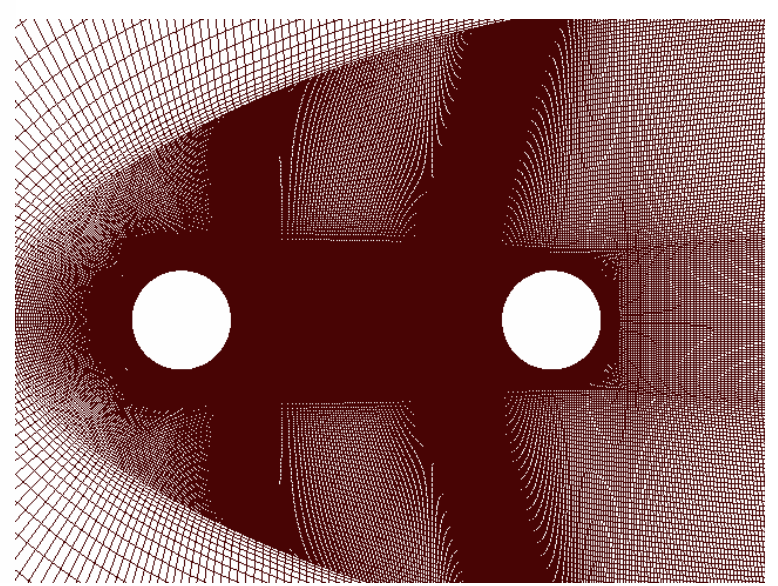

b) Near field grid

Fig.2. Computational domain and grid distribution ( $\mathrm{L} / \mathrm{D}=3.7)$ 


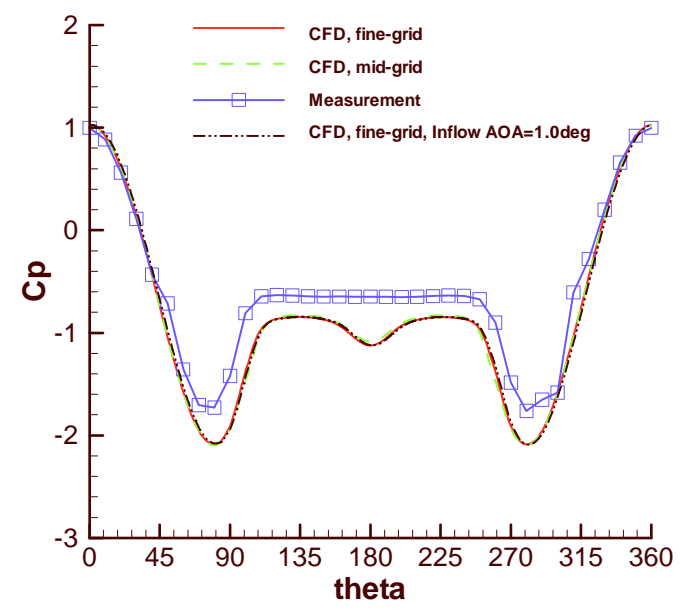

a) Front cylinder

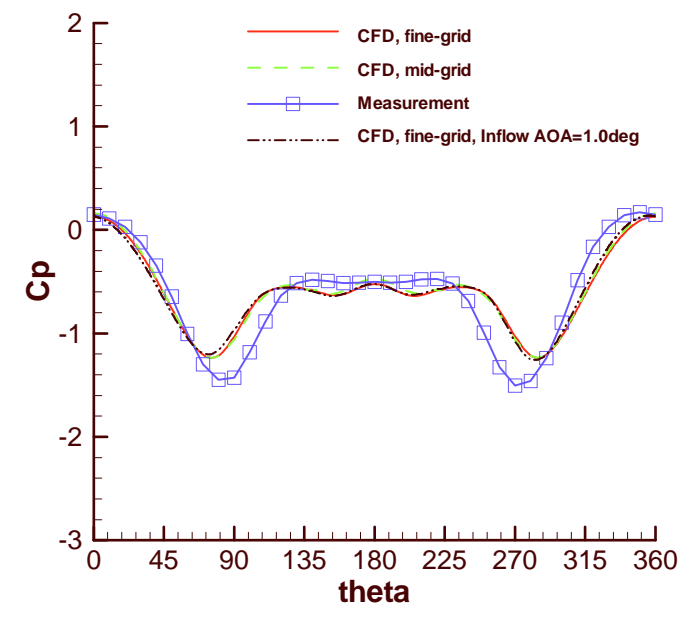

b) Rear cylinder

Fig.3. Surface pressure distribution for $\mathrm{L} / \mathrm{D}=3.7, \mathrm{M}=0.166$, and $\mathrm{Re}=0.166$ milion

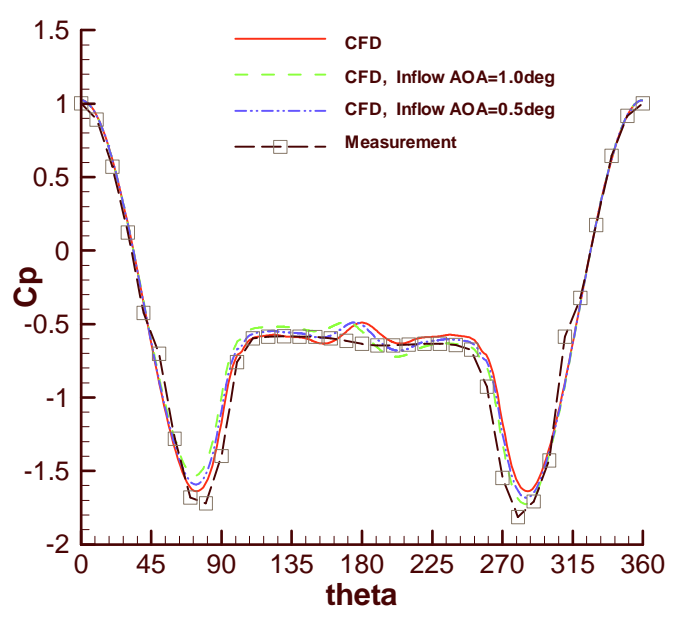

a) Front cylinder

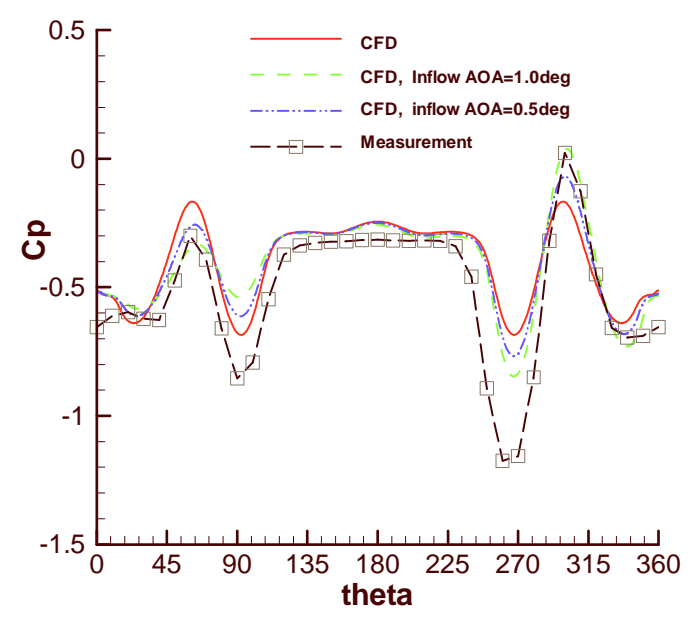

b) Rear cylinder

Fig.3. Surface pressure distribution for $\mathrm{L} / \mathrm{D}=1.435, \mathrm{M}=0.166$, and $\mathrm{Re}=0.166$ milion 


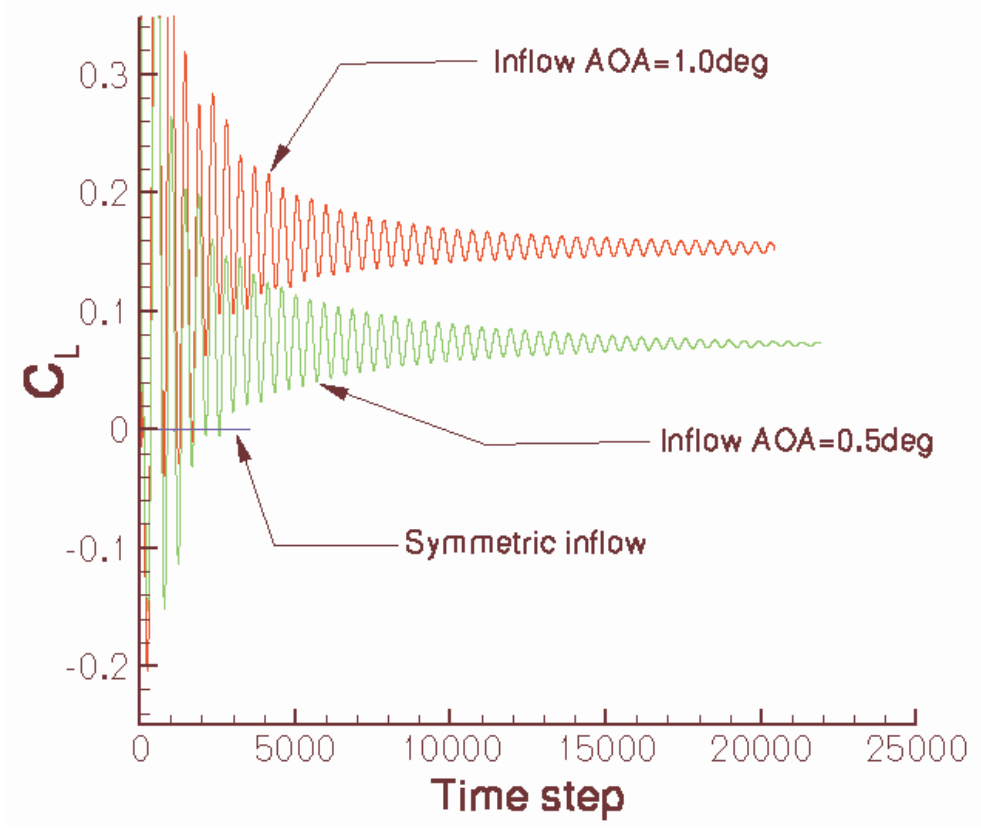

Fig. 5. Effect of incoming flow angularity on total lift for $L / D=1.435$

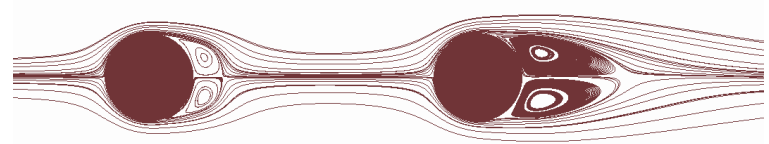

a) Computation

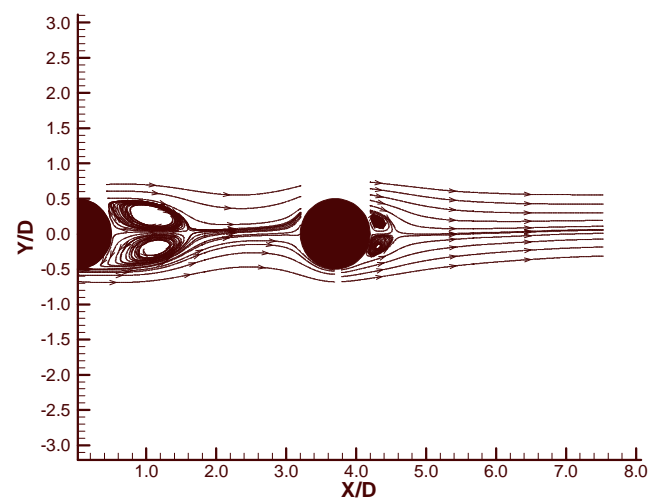

b) Experiment

Fig. 6. Mean streamlines for $\mathrm{L} / \mathrm{D}=3.7$ 


\section{Computation}

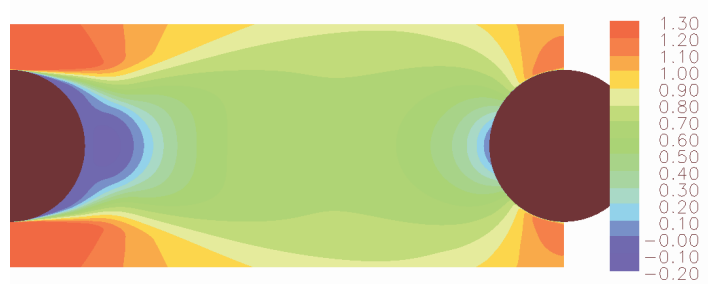

Fig. 7a. Streamwise velocity

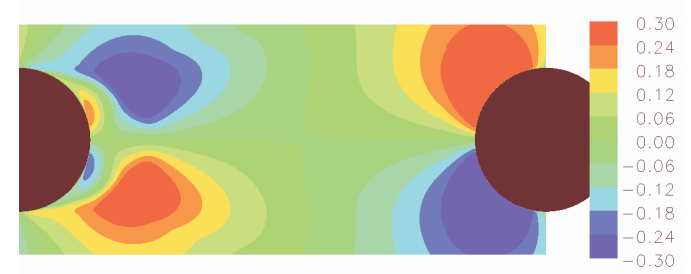

Fig. 7c. Vertical velocity

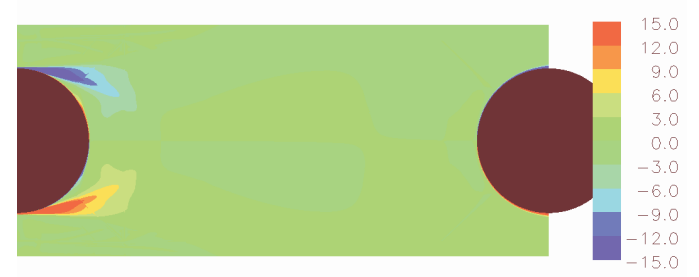

Fig. 7e. Spanwise vorticity
Experiment

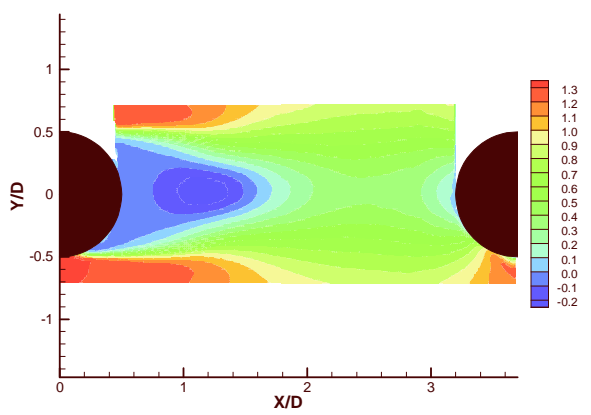

Fig. 7b. Streamwise velocity

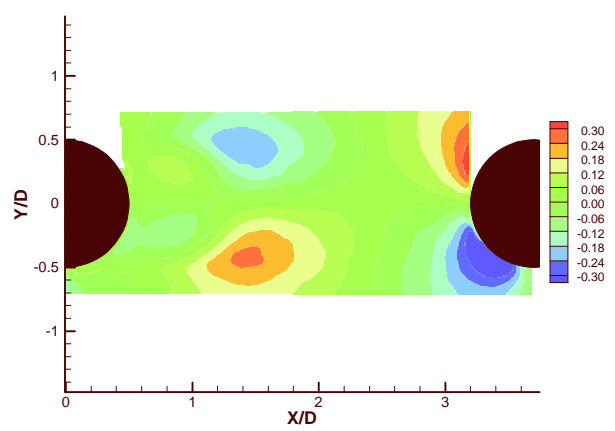

Fig. 7d. vertical velocity

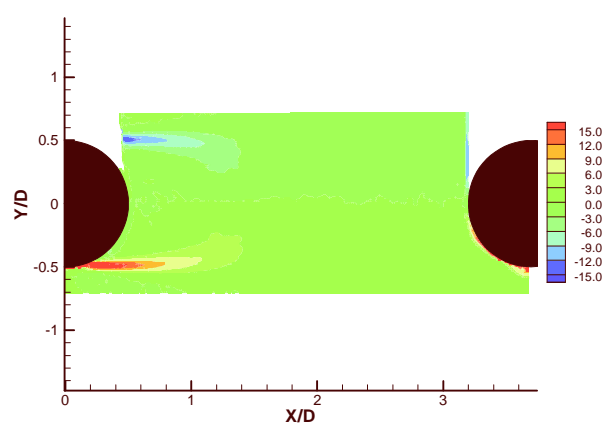

Fig. 7f. Spanwise vorticity

Fig. 7. Mean velocity and vorticity fields for $\mathrm{L} / \mathrm{D}=3.7, \mathrm{M}=0.166$, and $\mathrm{Re}=0.166$ million 


\section{Computation}

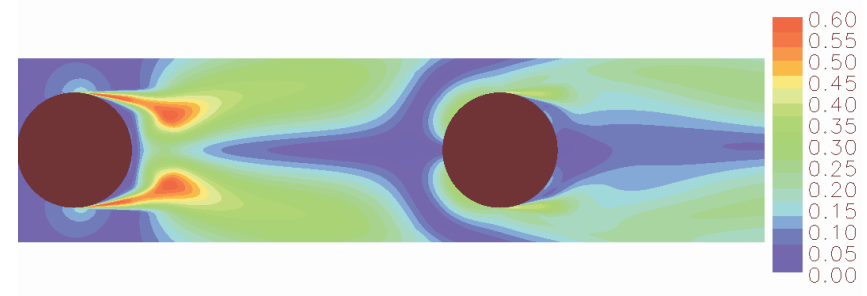

Fig. 8a. Streamwise velocity

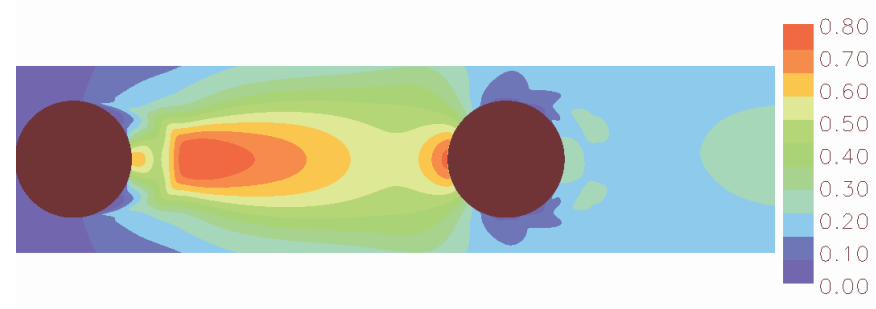

Fig. 8c. Vertical velocity

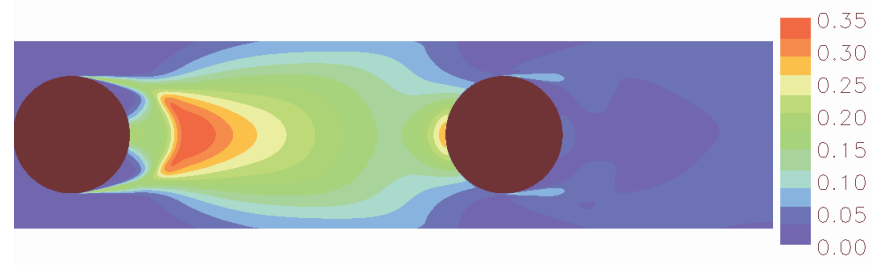

Fig. 8e. TKE
Experiment

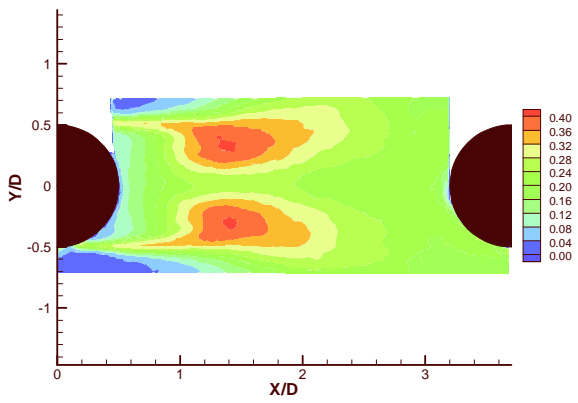

Fig. 8b. Streamwise intensities

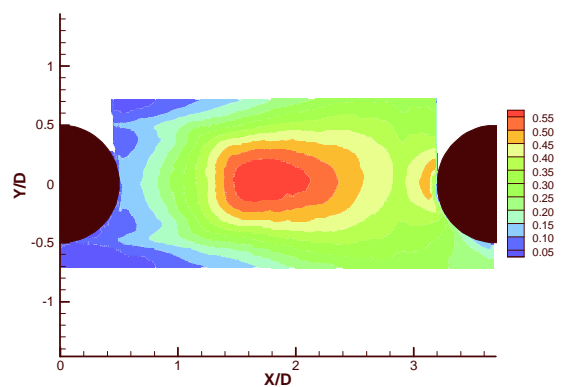

Fig. 8d. Vertical intensities

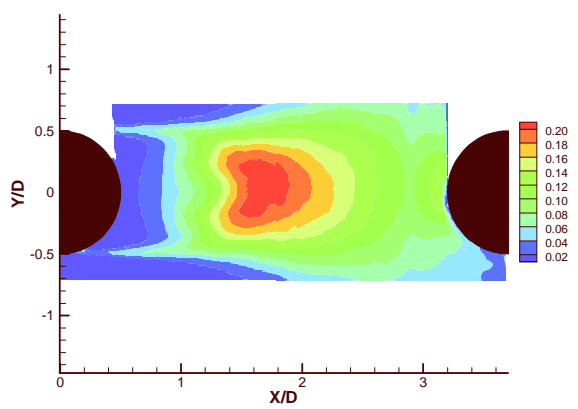

Fig. 8f. TKE

Fig. 8. Mean fluctuating field for $\mathrm{L} / \mathrm{D}=3.7, \mathrm{M}=0.166$, and $\mathrm{Re}=0.166$ million 


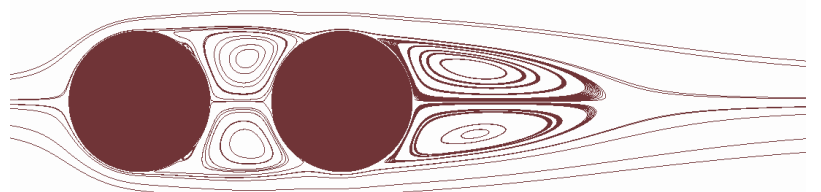

a) Computation

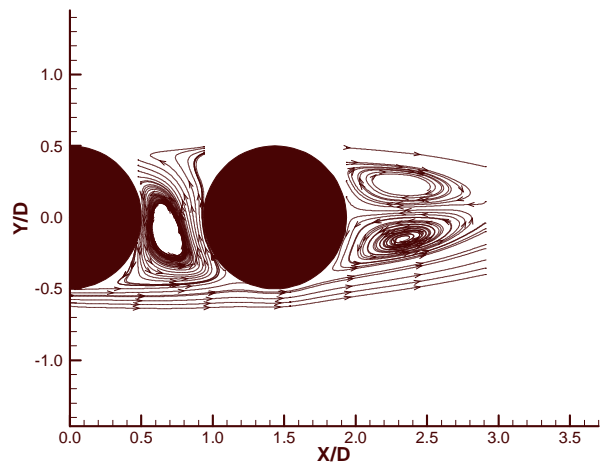

b) Experiment

Fig. 9. Mean streamlines for $\mathrm{L} / \mathrm{D}=1.435$ 
Computation

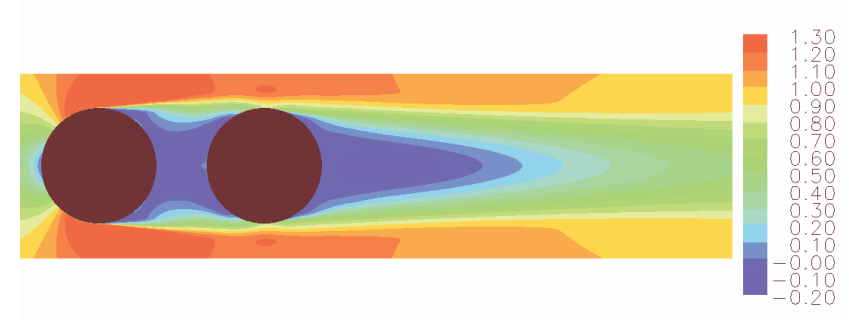

Fig. 10a. Streamwise velocity

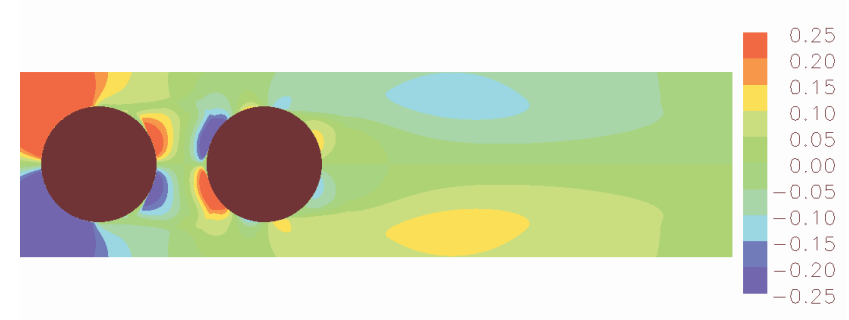

Fig. 10c. Vertical velocity

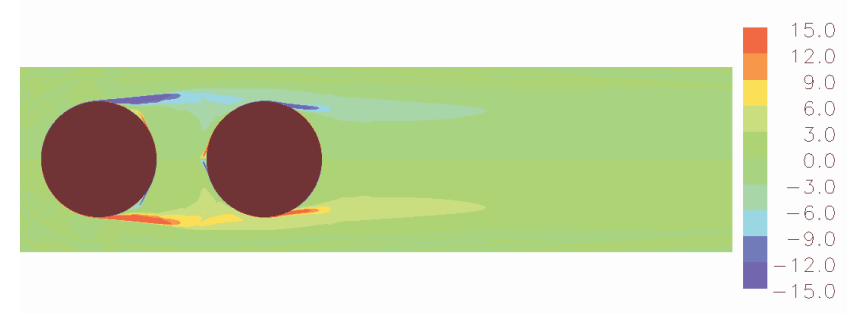

Fig. 10e. Spanwise vorticity
Experiment

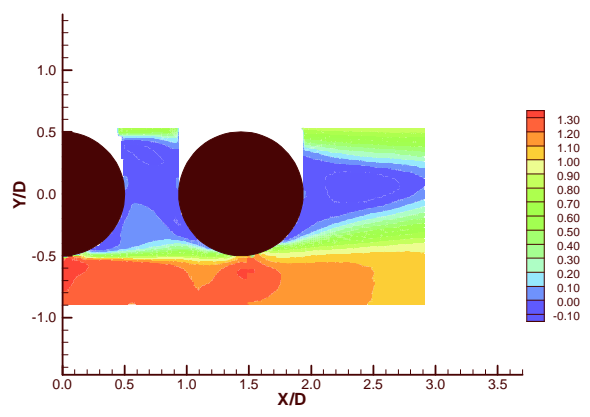

Fig. 10b. Streamwise velocity

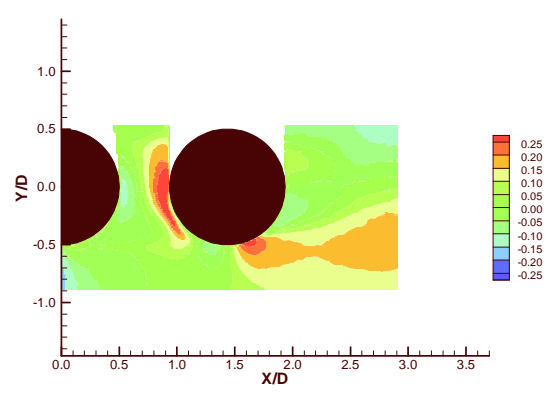

Fig. 10d. Vertical velocity

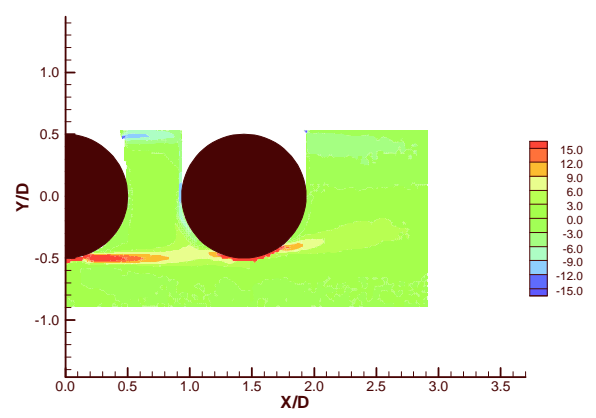

Fig. 10f. Spanwise vorticity

Fig. 10. Mean velocity and vorticity fields for $\mathrm{L} / \mathrm{D}=1.435, \mathrm{M}=0.166$, and $\mathrm{Re}=0.166$ million 


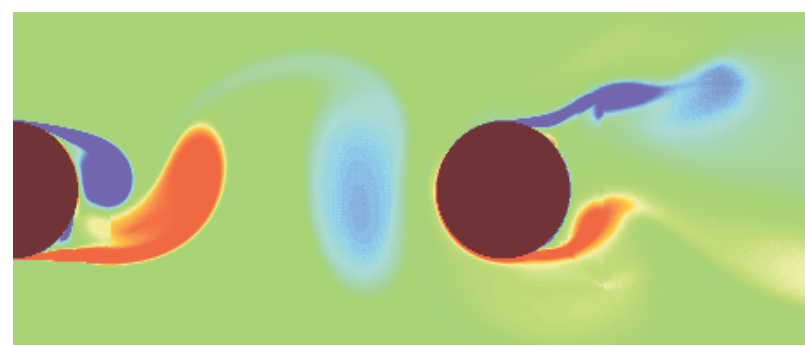

a) Computation

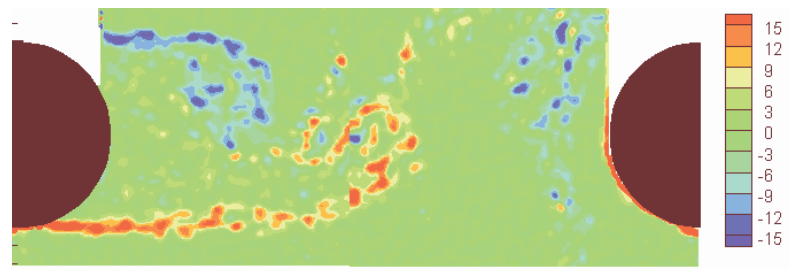

b) Experiment

Fig. 11. Instantaneous spanwise vorticity field for $\mathrm{L} / \mathrm{D}=3.7$

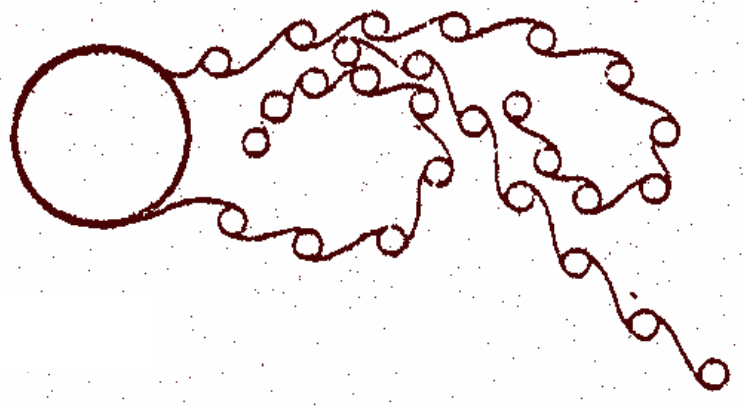

a)

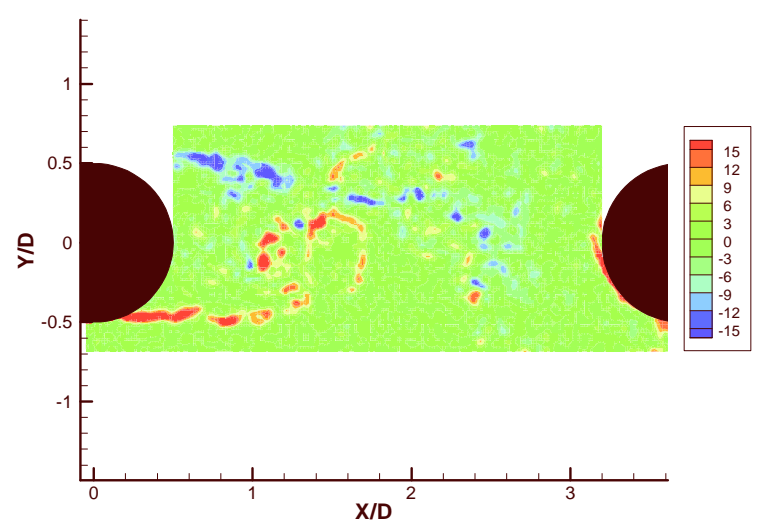

b)

Fig. 12. Schematic of wake structures as observed by Couregelongue (a) and a PIV snapshot of instantenous vorticiy field (b) showing similar structures 


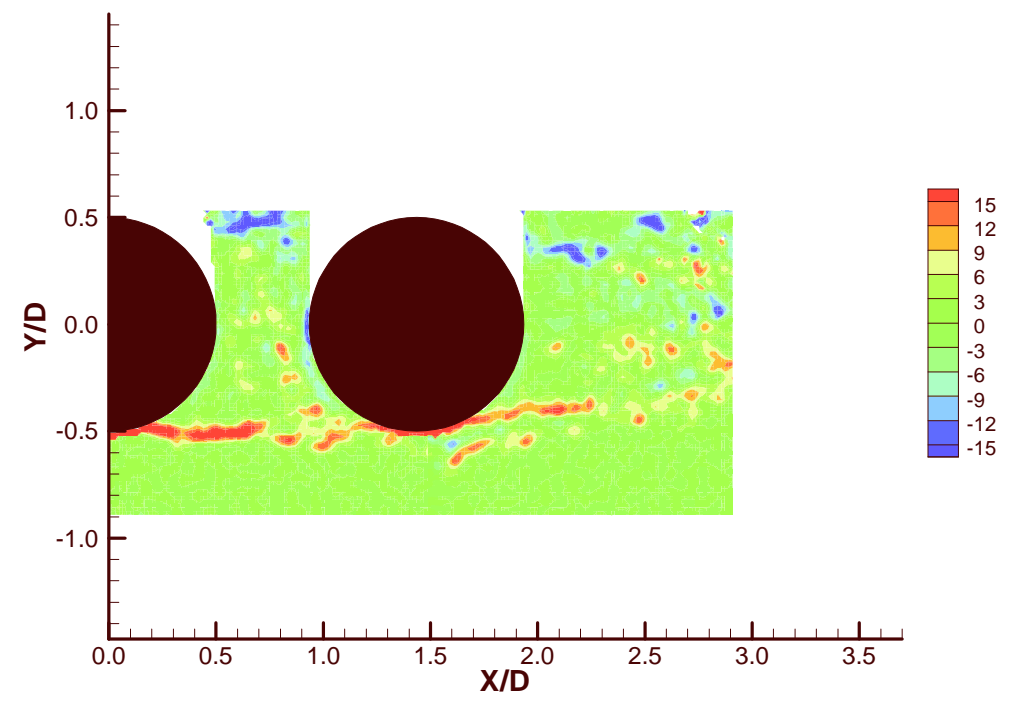

Fig. 13. Instantaneous spanwise vorticity for $\mathrm{L} / \mathrm{D}=1.435, \mathrm{M}=0.166$, and $\mathrm{Re}=0.166$ million

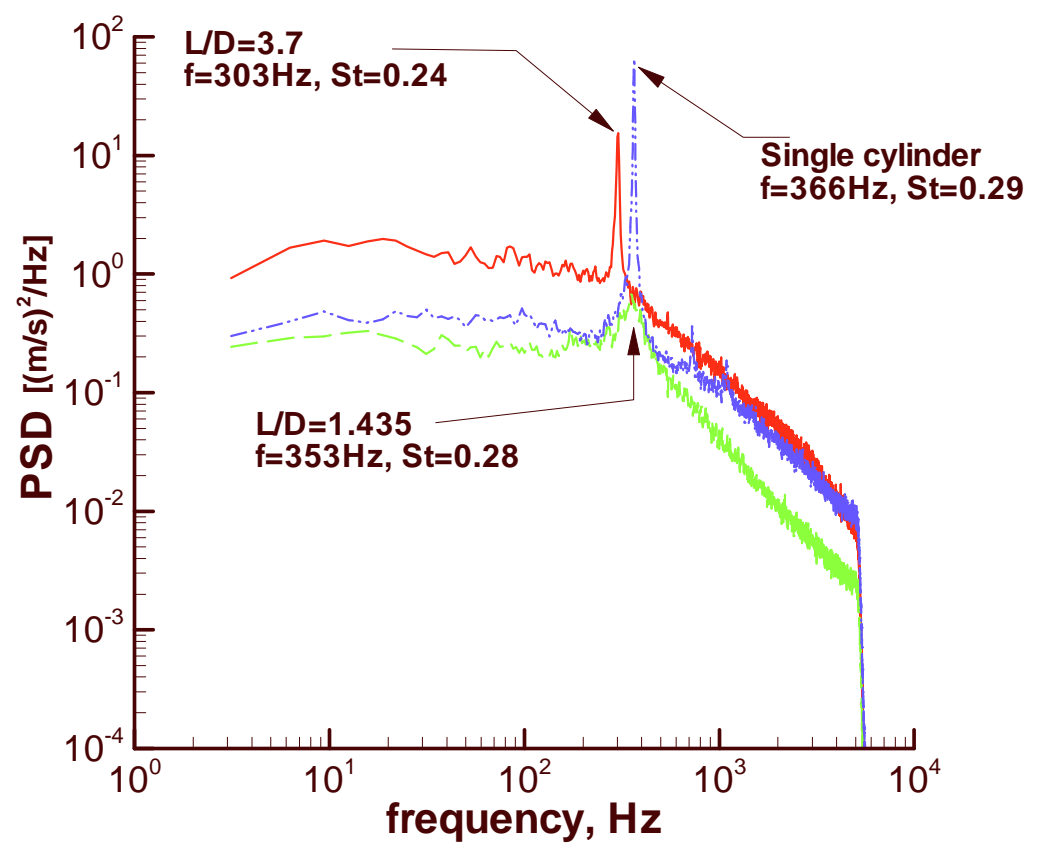

Fig. 14. Velocity fluctuation spectrum indicating shedding frequency, measured by hot-wire at a downstream streamwise distance of approximately2.7 diameters and $\mathrm{Y} / \mathrm{D}=-0.69$ behind the rear cylinder or single cylinder 


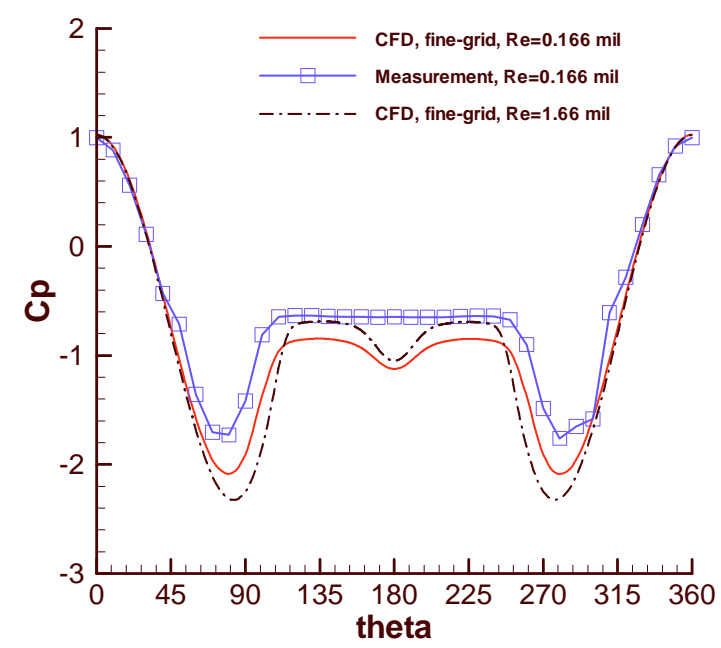

a) Front cylinder

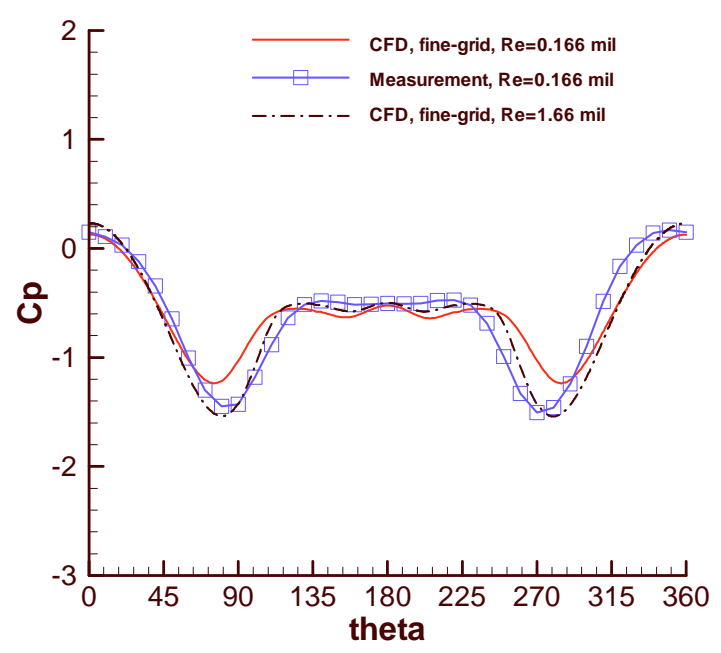

b) Rear cylinder

Fig. 15. Surface pressure distribution for $\mathrm{L} / \mathrm{D}=3.7, \mathrm{M}=0.166$, and $\mathrm{Re}=1.66$ million 